\title{
Recent Advancement in Human Reproduction Three-parent Babies: A Technique to Neutralize Mitochondrial Disease Load-A Boon or a Bane for Society?
}

\author{
${ }^{1}$ Simranpreet Kaur, ${ }^{2}$ Madhu Nagpal
}

\begin{abstract}
Three-parent baby technique is the latest tool in preventive methods for mitochondrial diseases. It uses the mitochondrial deoxyribonucleic acid (DNA) replacement therapy (MRT) to get rid of defective mitochondrial DNA (mtDNA). The MRT has been made possible by pronuclear transfer and maternal spindle transfer (MST). This shall take away the need of surrogacy and adoption, if practiced with certainty. The MRT replaces defective mtDNA, but its close functioning with the individual's nuclear DNA (nDNA) makes the whole process complicated. This article provides a summarized understanding of the biological mechanisms involved in MRT and the need to consider three-parent in vitro fertilization (IVF). It also discusses the bioethical issues of the technique considering its future implications and whether it is a boon or a bane for our society and subsequent generations.
\end{abstract}

Keywords: Bioethical issues, Germline genetic modification, Mitochondrial deoxyribonucleic acid, Mitochondrial replacement therapy, Three-parent in vitro fertilization.

How to cite this article: Kaur S, Nagpal M. Recent Advancement in Human Reproduction Three-parent Babies: A Technique to Neutralize Mitochondrial Disease Load-A Boon or a Bane for Society? Curr Trends Diagn Treat 2017;1(2):100-103.

Source of support: Nil

Conflict of interest: None

\section{INTRODUCTION}

In an era of advancement in science, research, and highdefinition technological assistance, the desire to achieve fertility is at its climax and that too of biologically natural offspring out of assisted reproduction. Prenatal preimplantation genetic diagnosis (PGD) is one step ahead in eliminating abnormal embryos. By conquering of the

${ }^{1}$ Embryologist and Counselor, ${ }^{2}$ Professor and Head

1,2Department of Obstetrics and Gynecology, Sri Guru Ram Das Institute of Medical Sciences \& Research, Amritsar, Punjab, India

Corresponding Author: Simranpreet Kaur, Embryologist and Counselor, Department of Obstetrics and Gynecology, Sri Guru Ram Das Institute of Medical Sciences \& Research, Amritsar Punjab, India, Phone: +911832870200 , e-mail: simranpreet sandhu@yahoo.com micromanipulative techniques the mitochondrial element has been maneuvered to achieve normal pregnancy in mitochondrial diseased subjects. This will take away the need for surrogacy and adoption, if practiced with certainty.

Mitochondrial diseases are a clinically heterogeneous group of disorders that result due to dysfunction of mitochondrial respiratory chains. ${ }^{1}$ The mitochondrion is a crucial eukaryotic organelle, which regulates cell energy production through oxidative phosphorylation.

Due to its role in energy metabolism, mitochondria are abundant in tissues with high-energy demands, such as in brain, heart, muscle, kidney, liver, and the central nervous system tissues. This unique feature is that it characterizes multiorgan involvement in mitochondrial genetic diseases, ${ }^{2}$ such as in Leigh's disease, mitochondrial myopathy, encephalopathy, lactic acidosis and strokelike episodes, myoclonic epilepsy with ragged red fibers, Pearson syndrome, short chain acyl coenzyme A dehydrogenase deficiency, etc. ${ }^{3}$ Mitochondrial genetic diseases are caused by mutations either in mtDNA or in the nuclear genes involved in mitochondrial function. In humans, mtDNA is maternally inherited since the father's mtDNA is located in the sperm midpiece, which is lost at fertilization ${ }^{4}$ or specifically removed by ubiquitination. ${ }^{5,6}$ The human oocytes have 100,000 copies of mtDNA, whereas the sperm contains only 100 copies. $^{7}$

Disruption of essential metabolic pathways in people suffering from mtDNA disease, mainly in high-energy demanding organs, leads to severe disability and early death. Clinical diagnosis is challenging due to varying symptoms and phenotypes even within a single family. This happens due to mitochondrial heteroplasmy, where mutational load at the cellular level determines phenotype. The low mutational load will result in asymptomatic phenotypes or low severity of the disease, but once the number of affected mitochondria exceeds a certain threshold, patients become increasingly symptomatic. Usually, there is unequal distribution of mutated mtDNA between organs depending on the mitochondrial segregation patterns during fetal development, thereby giving rise to various phenotypes depending on the levels of mutated mtDNA present and the organs involved. ${ }^{8}$ 
A large number of serious body malfunctions are caused by mitochondrial diseases including fatal ones. This debilitating genetic disorder can produce an increased risk of infection, auditory and visual impairment, disease of liver, heart, kidneys, many neurological and physiological problems that include seizures, loss of coordination, muscle weakness, diabetes, and stunned growth. This vast majority of symptoms usually affect the child before the age of 10 years, ${ }^{9}$ and once these dreadful diseases develop, there is no effective treatment for them. A woman who suffers from a mitochondrial disease or is a carrier for the same will certainly pass on her genetic material (both nDNA and mtDNA) to her offspring along with transmitting the disease. Moreover, a woman who gives birth to a child plagued by a mitochondrial disease is likely to repeat the transmission to possibly every one of her future children with $100 \%$ certainty. ${ }^{10}$

There came up a number of interventions for treatment of mitochondrial diseases, which includes vitamin supplements, various drugs, and physical exercise, but all of them proved to be ineffective in the treatment of mitochondrial disorders. ${ }^{11}$ The greatest hope came in the form of three-parent IVF, mainly known as mitochondrial replacement IVF, that seems to have great potential, as the women who are affected by disease due to an alteration in their mtDNA and the asymptomatic carriers of the disease can have children free from mutation. ${ }^{12}$

\section{THREE-PARENT IVF: MRT}

The exchange of nuclear genetic material between oocytes and embryos offers a novel reproductive option for the prevention of inherited mitochondrial diseases.

Two approaches have been proposed:

1. Pronuclear transfer or cytoplasmic transfer

2. Maternal spindle transfer

In pronuclear transfer, two zygotes are created in vitro. One is created with the intending parent's sperm and oocyte, the other one is created with a donated oocyte and an intended father's sperm. After fertilization and during the first 24 hours, the maternal and paternal pronuclei are removed from both zygotes. The enucleated cell produced with intended mother's oocyte and the pronuclei contained in the cell produced by donor's oocyte are discarded. Subsequently, the pronuclei of intended parents are transferred to the enucleated cell produced with donor's oocyte. The reconstructed zygote is then transferred to the intended mother or a surrogate. ${ }^{13}$

The other technique is MST in which through assisted reproductive techniques, eggs are obtained from the intended mother and a healthy donor. The nucleus from the intended mother's oocyte is taken and transferred into a healthy donor's oocyte whose own nucleus had been removed. The reconstituted oocyte contains all the normal genes from the mother, but her faulty mitochondria are replaced by those from the healthy donor. The reconstructed oocyte is then fertilized with the father's sperm and the embryo is transferred to the intended mother or surrogate. ${ }^{14,15}$

The resulting embryo from either of the above techniques has usual 23 pairs of chromosomes that hold the mother and father's DNA, but the 37 mitochondrial genes making $0.2 \%$ of the total came from the third person, the donor.

In year 2016, the world's first baby was born using one of the mitochondrial transfer techniques. The baby was born on April 6, 2016 to Jordanian parents, who traveled to Mexico where they were cared for by US Fertility specialists as three-parent IVF is banned in the United States. The baby boy's mother carries genes for the fatal Leigh syndrome, which harms the developing nervous system. The mother is healthy, but about one-quarter of her mitochondria carried genes for Leigh syndrome. The couple had already lost six children to mitochondrial heteroplasmy of the mother. Dr John Zhang ${ }^{16}$ from the New Hope Fertility Centre in New York came up with the clinical trial of MRT.

The second baby in the world was born on January 5 , 2017 with MRT using the pronuclear transfer method for the first time. The Nadiya clinic in Kiev, Ukraine, used the technique to treat an infertile couple. ${ }^{17}$

\section{Three-parent IVF: A Boon}

\section{Prevention of Mitochondrial Disease}

Mitochondrial replacement techniques will offer a therapeutic reproductive option for patients suffering from mitochondrial disease. Otherwise, women who are at a risk of transmitting mtDNA disease have to resort to donor oocyte or PGD technique, both of which have their own limitations. Use of donor oocytes results in the intended mother being genetically unrelated to the child. On the contrary, PGD is unsuccessful in detecting mtDNA mutations in women who carry high mutational load in oocytes, but low mutational load in cells outside ovaries. Moreover, PGD does not provide any preventive solution as if embryos attained are tested by PGD and found affected, therefore, they are untransferrable. As a result, it is important to focus on eliminating mutant mtDNA from the embryo, possibly by mitochondrial transfer. ${ }^{18}$

\section{Reviving Aged Ova}

Women have been postponing childbirth beyond 30 years of age consequently leading to reproductive decline. Female reproductive physiology has not kept up with 
recent social and cultural tendencies resulting in agerelated infertility, aneuploidy, and fetal defects in women of advanced maternal age. ${ }^{19}$ Major causative factors are mitochondrial dysfunction from accumulation of mtDNA mutations and accumulation of oxygen radicals. ${ }^{20}$ Mitochondria are the primary sources of intercellular production of reactive oxygen species (ROS). Increased ROS levels lead to decreased metabolic function and energy production. Continuous accumulation of mutations arising from age-related ROS also affects the potential of the mtDNA genome to replicate, thereby leading to aging. ${ }^{21}$

The mutation rate of mtDNA is fifteen times that of the nuclear genome. Moreover, mtDNA lacks protective histones, which makes it deficient in DNA repair mechanisms. ${ }^{22}$ Fertilization potential of oocytes and developmental potential of embryos are largely dependent on adenosine 5-triphosphate (ATP) and mtDNA content. Poor implantation leading to infertility also occurs due to low mtDNA copy number as implantation is an energydemanding process.

Hence, mitochondrial transfer could potentially overcome infertility issues via introduction of large amounts of healthy mitochondria, thereby supplying the necessary amount of ATP required for fertilization.

\section{Diabetic Mothers-to-Be}

Diabetic women mimic older mothers-to-be in their poor reproductive outcomes, experiencing increased rates of aneuploidies, miscarriages, and birth defects, which have been attributed to mitochondrial dysfunction. ${ }^{23}$ It has been believed that in diabetic patients, there is high oxidative stress leading to changes in mitochondrial ultrastructure, increased mtDNA copy number, and reduced levels of ATP and tricarboxylic acid cycle metabolites. ${ }^{24,25}$ Researchers also concluded that oocytes develop abnormally due to toxic diabetic environment, which triggers mitochondrial damage. ${ }^{26}$ There is a correlation between mitochondrial dysfunction and insulin resistance, thereby predisposing the offspring to obesity and insulin resistance, which are developing from oocytes containing defective mitochondria. By introducing the nuclear genome of the affected mother into healthy oocytes that possess undamaged mitochondria, diabetic women will have an improved chance of falling pregnant with healthy fetus.

\section{Three-parent IVF: A Bane}

The MRT has raised many bioethical concerns some of which are:

\section{Possible Destruction of Embryos}

The report of the Institute of Medicine to the US Food and Drug Administration states that "In addition to manipulation, MRT would involve the creation and possible destruction of embryos, both in the research phase and in clinical use. The ethical, social and policy concerns surrounding the creation and destruction of embryos are long standing. Religious, ethical, social, and policy issues are associated with the creation, manipulation, and destruction of human embryos." 27 The concern is about disposal of embryos. As to make one life, two embryos are discarded, which is against the Catholic Church. ${ }^{27}$

\section{Interconnection between nDNA and mtDNA}

There is a close interconnection between nDNA and mtDNA of an individual, which has become highly specific over evolutionary time. Many studies suggest that mtDNA variant in an individual has an effect on its interaction with nDNA, which affects the synthesis of mitochondrial proteins, oxidative stress, insulin signaling, obesity, parameters of aging that include telomere shortening and mitochondrial dysfunction, ${ }^{28}$ so the novel combinations of $\mathrm{nDNA}$ of an individual and $\mathrm{mtDNA}$ from donor occurring in MRT may be mismatched, leading to severe health complications in future generations.

\section{Making of Designer Babies}

The other ethical issue is about making of "designer babies" through MRT as it is a germline genetic modification and the fear is that it will provide scientists with "a quiet way station" in which to refine the micromanipulation techniques essential for other human germline interventions (including nDNA germline modification) and human cloning, ${ }^{29}$ which in the end will lead to "designer babies."

\section{mtDNA Carryover}

The first two babies with donor mtDNA have already been born through different techniques of MRT, but the safety concerns about the future health of children are still unresolved. There is no clear-cut surety of how much of mtDNA carryover has taken place in the babies from their affected mother, because the mutant mtDNA levels increase during subsequent development of the baby and disease reappears in later generations. ${ }^{11}$

\section{Lack of Regulation Laws}

Because MRT is in its initial stage, there are no regulation laws so far, which can take care of mtDNA donor's well-being and which protect them from negative effects of repeated ovarian hyperstimulation. ${ }^{15}$

\section{CONCLUSION}

Though MRT is going to be an excellent tool for decreasing mitochondrial disease load from society, to develop it 
as a wholesome preventive method, we have to consider its social, ethical, religious, and physiological aspects. For this, a large amount of research is still needed in the area of MRT. More studies need to be conducted in animal models to study mitochondria-nucleus communication, transmission of mtDNA to the offspring-especially in regard to the variable transmission of heteroplasmy. An in-depth understanding of the biological mechanisms involved is still needed to consider if three-parent IVF is a boon or a bane for our society and for the future generations.

\section{REFERENCES}

1. Chinnery PF. Mitochondrial Disorders overview. In: Adam MP, Ardinger HH, Pagon RA, Wallace SE, Bean LJH, Stephens K, Amemiya A, editors. GeneReviews. Seattle (WA): University of Washington; 2000. [cited 2014 Aug 14]. Available from: http:/ / www.ncbi.nlm.nih.gov/pubmed/20301403.

2. Lui H-S, Chu P-L. Three parent embryo: the therapeutic future for inherited mitochondrial diseases. J Formos Med Assoc 2015 Nov;114(11):1027-1028.

3. UMDF. The mitochondrial disease community registry. [cited 2017 Apr 5]. Available from: www.umdf.org.

4. Paternal mtDNA transmission. [cited 2017 Apr 8]. Available from: http://en.wikipedia.org/wiki/Paternal_mtDNA_ transmission.

5. Carelli V. Keeping in shape the dogma of mitochondrial DNA maternal inheritance. PloS Genet 2015 May;11(5):e1005179.

6. Sutovsky P. Ubiquitin-dependent protolysis in mammalian spermatogenesis, fertilization and sperm quality control: killing three birds with one stone. Microsc Res Tech 2003 May;61(1):88-102.

7. Larsson NG, Clayton DA. Molecular genetic aspects of human mitochondrial disorders. Annu Rev Genet 1995 Dec;29: 151-178.

8. Border P. Preventing mitochondrial disease. Post note 431. London: Parliamentary Office of Science and Technology of the UK Parliament; 2013. Available from http:/ / www.parliament.UK/business/publications/research/briefingpapers / POST-PN-43/preventing_mitochondrial_disease.pdf.

9. Taylor RW, Turnbull DM. Mitochondrial DNA mutations in human disease. Nat Rev Genet 2005 May;6(5):389-402.

10. Garasic MD, Sperling D. Mitochondrial replacement and parenthood. Global Bioethics 2015 Jul;26(3-4):198-205.

11. Gómez-Tatay L, Hernández-Andreu JM, Aznar J. Mitochondrial modification techniques and ethical issues. J Clin Med 2017 Feb;6(3):E25.

12. Amato P, Tachibana M, Sparman M, Mitalipov S. Three parent in vitro fertilization: gene replacement for the prevention of inherited mitochondrial diseases. Fertil Steril 2004 Jan;101(1):31-35.

13. Craven L, Tuppen HA, Greggains GD, HarbottleSJ, Murphy JL, CreeLM, MurdochAP, Chinnery PF, Taylor RW, Lightowlers RN, et al. Pronuclear transfer in human embryos to prevent transmission of mitochondrial DNA disease. Nature 2010 May;465(7294):82-85.
14. Tachibana M, Sparman M, Sritanaudomchai H, Ma H, Clepper L, Woodward J, Li Y, Ramsey C, Kolotushkina O, Mitalipov S. Mitochondrial gene replacement in primate offspring and embryonic stem cells. Nature 2009 Sep;461(7262): 367-372.

15. Hallowell N. Nuffield Council on Bioethics: novel techniques for the prevention of mitochondrial DNA disorders: an ethical review. Genom Soc Policy 2012 Dec;8(2):29-31.

16. Zhang J. World's first baby born from new procedure using DNA of three people. New York: New Hope Fertility Center; 2016. [cited 2017 Mar 10]. Available from: http://www. theguardian.com/science/2016/sept/27/worlds-first-babyusing-dna-from-three-parents.

17. Roberts M. IVF: first three-parent baby born to infertile couple. BBC News Online; 2017. [cited 2017 Mar 11]. Available from: www.bbc.com/news/health-38648981.

18. Reznichenko AS, Huyser C, Pepper MS. Mitochondrial transfer: implications for assisted reproductive technologies. Appl Transl Genom 2016 Dec;11:40-47.

19. Bentov Y, Yavorska T, Esfandiari N, Jurisicova A, Casper RF. The contribution of mitochondrial function to reproductive ageing. J Assist Reprod Genet 2011 Sep;28(9):773-783.

20. Reynier P, May-Panloup P, Cheretien MF, Morgan CJ, Jean M, Savagner F, Barriere P, Malthiery Y. Mitochondrial DNA content affects the fertilizability of human oocytes. Mol Hum Reprod 2001 May;7(5):425-429.

21. Kirkwood TB. Understanding the odd science of aging. Cell 2005 Feb;120(4):437-447.

22. MecocciP,FanóG,FulleS,MacGarveyU,ShinobuL,PolidoriMC, Cherubini A, Vecchiet J, Senin U, Beal MF. Age dependent increases in oxidative damage to DNA, lipids and proteins in human skeletal muscle. Free Radic Biol Med 1999 Feb;26(3-4): 303-308.

23. Maritim AC, Sanders RA, Watkins JB 3rd. Diabetes, oxidative stress and antioxidants: a review. J Biochem Mol Toxicol 2003 Feb;17(1):24-38.

24. Bentov Y, Casper RF. The aging oocyte-Can mitochondrial function be improved? Fertil Steril 2013 Jan;99(1):18-22.

25. Calabrese V, Lodi R, Tonon C, D'Agata V, Sapienza M, Scapagnini G, Mangiameli A, Pennisi G, Stella AM, Butterfield DA. Oxidative stress, mitochondrial dysfunction and cellular stress response in Friedreich's ataxia. J Neurol Sci 2005 Jun;233(1-2): 145-162.

26. Wang Q, Ratchford AM, Chi MM, Schoeller E, Frolova A, Schedl T, Moley KH. Maternal diabetes causes mitochondrial dysfunction and meiotic defects in murine oocytes. Mol Endocrinol 2009 Oct;23(10):1603-1612.

27. National Academies of Sciences, Engineering and Medicine. Mitochondrial Replacement Techniques: Ethical, Social and Policy considerations. Washington (DC): National Academies Press; 2016. Available from: http:/ / www.nationalacademies. org/hmd/ /media/Files/Report\%20Files/2016/Mitochondrial\%20Replacement\%20Techniques/MitoEthics-RIB.pdf.

28. ReinhardtK, Dowling DK, Morrow EH. Mitochondrial replacement, evolution, and the clinic. Science 2013 Sep;341(6152): 1345-1346.

29. Baylis F. Human nuclear genome transfer (so-called mitochondrial replacement): clearing the underbrush. Bioethics 2017 Jan;31(1):7-19. 